\title{
Retos y oportunidades de la tecnología móvil en la educación física Challenges and opportunities of mobile technology in physical education
}

\author{
José Díaz Barahona
}

Universidad de Valencia (España)

\begin{abstract}
Resumen. La educación física (EF) y su profesorado están llamados a seguir procesos de inmersión digital, voluntarios o involuntarios, asociados al consumo masivo de medios y servicios digitales y a las demandas de la sociedad y de la institución educativa. El uso de tecnología, especialmente la móvil, social y ubicua, se generaliza personal y profesionalmente, aunque la escuela sigue sin encontrarle acomodo. Reconoce su potencial didáctico, pero no logra alcanzarlo. En la EF el uso de tecnologías tradicionales y fijas han demostrado su utilidad en la fase preactiva y postactiva del acto didáctico, sin embargo, los aprendizajes en movilidad propios de la fase activa, aconsejan explorar alternativas como las que ofrece el mobile learning. El objetivo del artículo es mostrar el potencial de mejora e innovación pedagógica que tiene el mobile learning y las aplicaciones (Apps) en EF y proporcionar a los docentes e investigadores un marco de análisis que muestre cómo conseguirlo. Las aportaciones teóricas son necesarias, pero no suficientes, por ello se ofrecen orientaciones concretas que explican cómo y para qué integrar los dispositivos móviles en la EF. También se proponen futuras líneas de debate, reflexión e investigación centradas en la dimensión pedagógica de aprendizaje móvil, analizando las repercusiones éticas, sociales y legales derivadas de los procesos de inmersión digital.
\end{abstract}

Palabras clave: Apps, dispositivos móviles, educación física, mobile learning, seguridad y protección datos.

\begin{abstract}
Physical education (PE) and its teachers are destined to voluntarily or involuntarily follow processes of digital immersion associated with the mass consumption of digital media and with the current demands of both society and educational institutions. The use of mobiles, which is a social and omnipresent technology, is being increasingly generalized in both personal and professional areas of life; however, mobile use is virtually non-existent in the field of education. Educational institutions and teachers recognize the educational potential of mobile technology yet fail to implement its use. The use of office technologies in PE has already shown its value in both the preactive and proactive phases of the educational process, however, the kinetic learning processes associated to the active phase would benefit from alternatives such as mobile learning. The aim of the present article is to show the educational potential of mobile learning and to provide educators, researchers, and Apps developers with a framework outlining how to achieve it. Theoretical insights are important, but not sufficient. That is why orientations and concrete proposals are offered, explaining how and to what end should mobile devices be integrated into PE. Future debates, reflections, and research issues are also raised, focusing on the pedagogical dimension of mobile learning, and analyzing the ethical, social, and legal repercussions arising from the process of digital immersion.
\end{abstract}

Key Words: Apps, mobile devices, physical education, mobile learning, security and data protection.

\section{Introducción y contextualización del tema}

Los dispositivos móviles, totalmente integrados en nuestras vidas, están llamados a cambiar la forma de enseñar y de aprender, aunque por ahora los educadores, también los educadores físicos, sigan teniendo más expectativas que resultados (Casey, Goodyear \&Armour, 2017; Díaz, 2018; Robinson \& Aronica, 2015). Integrar las tecnologías de la información y la comunicación (TIC) en las aulas está resultando un proceso complejo y contradictorio, prueba de ello es que todavía no se ha conseguido (Aubusson, Schuck \& Burden, 2016). Sirvan las reflexiones de este apartado inicial para ubicar y justificar la relación entre el aprendizaje móvil (mobile learning) y la EF.

En educación física y deportiva, pese a la actitud positiva y pretecnológica de su profesorado y su aceptable competencia digital (CD), se confirma la existencia de barreras de uso (Can, 2015; Díaz, 2015; Wyant \& Baek, 2018) originadas por la falta de dispositivos y servicios móviles o por la mala ubicación de los recursos disponibles; generalmente TIC tradicionales alejadas de los espacios de aprendizaje (Díaz, 2015; Gibbone, Rukavina \& Silverman, 2010). Esta dificultad para usar la tecnología tradicional, fija y expositiva, especialmente durante las clases, invita a explorar nuevas alternati-

Fecha recepción: 24-11-18. Fecha de aceptación: 04-07-19 José Díaz Barahona jose.diaz-barahona@uv.es vas como las que ofrece el mobile learning; especialmente ahora que el uso de teléfonos inteligentes (smartphones) y tabletas se ha generalizado y se dispone de abundante hardware y software específico (Crawford \& Fitzpatrick, 2015; Jacinto, 2018; Silva \& Montañez, 2019).

El trabajo no pretende realizar un detallado análisis conceptual sobre aprendizaje móvil, pero se considera oportuno, como señala Traxler (2007), contar con la credibilidad de una base conceptual como punto de partida. Para suscribir una definición de aprendizaje móvil que se adapte a la singularidad de la EF, la literatura señala dos grandes tradiciones: una de carácter tecnocéntrica y otra de carácter tecnopedagógica (Dennen \& Hao, 2014; Peng, Su, Chou \& Tsai, 2009). Desde este trabajo se suscribe la línea de ElHussein \& Cronje (2010) que conciben el mobile learning como «cualquier tipo de aprendizaje que tiene lugar en entornos y espacios de aprendizaje que tienen en cuenta la movilidad de la tecnología, la movilidad de los estudiantes y la movilidad del aprendizaje» (p.20). El énfasis focalizado en la movilidad del alumnado, sus experiencias y los espacios de prácticas, más que en concepciones centradas en los propios dispositivos móviles (Traxler, 2007), se ajusta a los objetivos de la EF. Así entendido, el mobile learning puede ser un valioso instrumento pedagógico para el aprender haciendo y reflexionando «en», «para» y «sobre» la acción, donde usuarios y usos tecnológicos se complementan.

Otra variable contextual importante que determina el potencial educativo del mobile learning, es el perfil del alumnado. La juventud en edad escolar o universitaria se viene identifi- 
cando generacionalmente con acepciones tecnológicas: Digital natives (Prensky, 2011), Residentes digitales (White \& Le Cornu, 2011), Generación Hashtag (Feixa, FernándezPlanells \& Figueras-Maz, 2016) o Generación App (Gardner \& Davis, 2014), como consecuencia de la tecnologización de sus vidas. Son jóvenes que no han conocido un mundo sin la ubicuidad de los medios digitales ni tienen conciencia de cómo era la vida sin internet, smartphones o redes sociales (Gardner \& Davis, 2014). Una generación, indican Feixa, Fernández-Planells \& Figueras-Maz (2016), hiperconectada y multimedial que recibe y procesa mucha información -dentro y fuera de la escuela- y tiene capacidad para realizar varias tareas simultáneamente (multitasking skills). Con independencia del cuño digital con el que asociemos al alumnado, lo importante no es que vivan rodeados de likes, followers o Whatsapp, permanentemente conectados con sus tabletas o smartphones. Lo esencial es el impacto cultural, intelectual y social que esas tecnologías están teniendo pues configuran su visión de la vida y del mundo (Feixa, Fernández-Planells \& Figueras-Maz, 2016). Como destacan Gardner \& Davis (2014), la tecnología móvil, social y ubicua, está perfilando esta generación y las venideras y configurando su identidad personal, intimidad o forma de socializarse y de aprender.

Por ello, si las tecnologías y aplicaciones móviles condicionan los aprendizajes y las experiencias vitales del alumnado, la institución educativa y su profesorado necesitan readaptar su discurso y sus métodos pedagógicos si quieren inspirar y formar a los jóvenes en el futuro (UNESCO, 2016). Los educadores físicos no son la excepción y pese a las dificultades y ciertas reticencias del colectivo (Díaz, 2015) deberían asumir esa realidad y aprovechar el potencial transformador del aprendizaje móvil.

El problema y la complejidad de la integración del mobile learning en la educación físico-deportiva, también se entiende analizando la contradicción entre la posesión de medios digitales y su resistencia a usarlos (Díaz, 2015; Loureiro, 2012). Así, Garmendia-Larrañaga, Jiménez-Iglesias, Casadodel-Río \& Mascheroni, (2016) confirman que «en los últimos cinco años se aprecia que en los centros escolares ha habido profundas modificaciones» (p.93), y señalan la paradoja. Por una parte, el 97\% del alumnado de 15-16 años dispone de smatphone con el que accede diariamente a internet desde multiplataformas: smartphones (59\%), portátiles (32\%) y tabletas (26\%), sin embargo, solo al 16\% del alumnado se le permite utilizar dispositivos móviles. Cabe preguntarse por qué la escuela veta el uso de dispositivos móviles y por qué los sigue considerando elementos procastinadores y disruptores, en lugar de integrarlos como instrumentos de aprendizaje. Díaz (2018), sugiere que más que un problema tecnológico existe un problema de formación, de uso o de conducta y señala que el único inconveniente que plantea un dispositivo móvil es no saber utilizarlo. En todo caso, lo que debería vetar o sancionar la escuela son los usos inadecuados de la tecnología, no la propia tecnología (Loureiro, 2012).

La institución escolar y su profesorado están sujetos a procesos, voluntarios o involuntarios, de inmersión tecnológica asociados al consumo masivo de medios y servicios digitales móviles y a una cultura digital del alumnado en constante evolución (Díaz, 2018; Gardner \& Davis, 2014). En este contexto, indica Traxler (2016), es fácil defender que los dispositivos y apps móviles deben integrarse en las aulas. Lo difícil es concretar cómo. Para responder a esta cuestión, el trabajo se propone como objetivo, analizar, desde una perspectiva pedagógica y crítica, cómo integrar el mobile learning y las apps en la EF, reflexionando sobre las derivas éticas, legales y sociales de estos procesos de inmersión digital.

\section{Tecnología móvil para los aprendizajes en acción}

¿Necesita la misma tecnología el profesorado y el alumnado de filosofía o de matemáticas que el de educación física? ¿Para qué sirve la tecnología cuando está anclada en lugares fijos alejada de los espacios de aprendizaje y acción? Estas y otras cuestiones deben ser analizadas por el profesorado ya que el momento pedagógico, la naturaleza de las prácticas y los escenarios de aprendizaje, junto a la actitud de los docentes, facilitan o dificultan la integración de las TIC (Crawford \& Fitzpatrick, 2015; Díaz, 2018).

Respecto al momento pedagógico y los escenarios de las prácticas de EF, Díaz (2018) confirma que en el fase preactiva y post-activa del acto didáctico -antes y después de las clases-, el profesorado viene haciendo un uso productivo de la tecnología tradicional para diseñar, gestionar o evaluar los contenidos. Sin embargo, esta tecnología de escritorio, pensada para ser utilizada en espacios cerrados y estables como las aulas teóricas, no resulta adecuada para gestionar las experiencias didácticas realizadas en espacios al aire libre, en patios o en pistas polideportivas. Ambientes de EF, como indica Kretschmann (2017), «antipáticos» pues no disponen de medios ni servicios digitales (hardware o conectividad, alimentación eléctrica), a pesar ser el lugar donde el alumnado y el profesorado comparten conocimiento y acción. La ausencia de medios y servicios digitales en los escenarios de práctica impiden su aprovechamiento (Crawford \& Fitzpatrick, 2015; Díaz, 2015) y como sugieren Aubusson, Schuck \& Burden (2016), conviene llevar las TIC a los espacios de acción y no a la inversa como viene ocurriendo.

En referencia al profesorado y los aprendizajes en movilidad, Aubusson, Schuck, \& Burden (2016), indican que:

[...] los maestros son itinerantes. No tienden a estar ubicados en un solo lugar, sino que se mueven entre las aulas, los patios de juego y las salas de profesores. La naturaleza de sus contextos laborales sugiere que el aprendizaje móvil sería la forma más adecuada para que se produzca el aprendizaje[...] (p. 234).

Traxler (2016), destacando las posibilidades del aprendizaje móvil, lo describe como una forma de cambiar la naturaleza de los aprendizajes porque «permite crear, poseer, transformar, debatir, descartar, compartir ideas y crear y transformar identidades y comunidades» (p.1); idea en la que abundan Lavay, Sakai, Ortiz \& Roth (2015) señalando que, con una simple tableta, se puede generar conocimiento en cualquier momento y lugar. Si entendemos el aprendizaje móvil como la acción complementaria de prácticas y metodologías de enseñanza apoyadas por dispositivos móviles, coincidiremos en que esta modalidad de aprendizaje puede convertirse en un instrumento apropiado para impartir la EF ya que permite al alumnado y al profesorado reflexionar «en», «para» 
y «sobre» la acción (Schön, 987), objetivo esencial de la EF. Por otra parte, las buenas prácticas con TIC demuestran que las tecnologías fijas y las móviles nos son incompatibles sino complementarias. Ambas tienen su propio contexto de uso y su función pedagógica. Para Díaz (2018), las TIC tradicionales y expositivas facilitan la adquisición de conocimiento teóricos, pero las móviles tienen mayor capacidad para producir conocimiento práctico. Sin embargo, la tecnología que mejor se adapta al movimiento de los cuerpos, los grandes espacios y los aprendizajes en acción, es la móvil, ubicua e inalámbrica.

\section{El desafío de buscar y seleccionar Apps para la Educa- ción Física y Deportiva}

Cherner, Dix \& Lee (2014) sugieren que aunque el profesorado y el alumnado utilizan apps a diario, entender qué son, cómo evolucionan y saber encontrarlas, nos ayuda a relacionarnos funcionalmente con ellas. Técnicamente una aplicación móvil (application, apli o app) es un software ejecutable en un dispositivo móvil que, dependiendo del funcionamiento pueden ser: Apps nativas, desarrolladas para un sistema operativo específico (p.e., iOS, Android Windows Phone, BlackBerry OS); Web Apps, desarrolladas en el lenguaje de programación propio de la web y, Web Apps nativas, un híbrido entre las dos anteriores. Las encontramos en repositorios oficiales como Google Play Store, App Store de iOS o en plataformas como Amazon AppStore, Windows Store, Opera Mobile Appstore o BlackBerry World, entre otras. También en bases de apps educativos, clasificadas por materias, edad o función educativa, como: Eduapps (http://eduapps.es/), AppCrawlr (http://appcrawlr.com/app/ ), Edshelf (https://edshelf.com/) u otras como Graphite.org (http://www.graphite.org), Apps4Edu (http://www.uen.org/ apps4edu/) o Appitic (http://www.appitic.com). Existen webs de EF como Physical Education Central (https://goo.gl/ qbrtFo) que ofrecen ejemplos de su utilidad didáctica.

En la actualidad, seleccionar una app constituye un desafío abrumador para el profesorado porque existen millones (Lindl, 2017); decenas de miles vinculadas con el fitness, la salud o la práctica de actividad físico-deportiva (Statistic Brain, 2016; Yu, Kulinna \& Lorenz, 2018), y los repositorios y tiendas de apps proporcionan información poco organizada (Cherner, Dix \& Lee, 2014; Cummiskey, 2013). Para simplificar su búsqueda y selección, Cherner, Dix \& Lee (2014) recomiendan consultar bases de datos educativas, rastrear redes sociales, webs especializadas en análisis y métricas de descargas (p.e. App Figures, App Trace, App Annie) o refinar con el navegador los términos relacionados con contenidos deseados. Otros criterios e indicadores sugeridos por la literatura (p.e. Cherner, Dix \& Lee, 2014; Díaz, 2018; Papadakis \& Kalogiannakis, 2017) para elegir apps de calidad son:

- Que la app identifique al desarrollador (persona, institución o empresa), preferentemente vinculado y competente en EF y que ofrezca una dirección de contacto, web o servicio de asistencia con tutoriales de aprendizaje y consejos para resolver dudas.

- Que la app sea adecuada al nivel de maduración, comprensión y a la edad del alumnado: para enseñanza obligatoria (6-16 años), se sugiere una valoración/clasificación, en la
Play Store de PEGI 3, y en la App Store de 4+. El profesorado debe revisarla y verificar que no contiene información inadecuada para el contexto educativo: datos sexistas, racistas, imágenes violentas o incite a comportamientos incívicos.

- La valoración y las reseñas de los clientes sobre una app son indicadores de calidad y fiabilidad, por lo que conviene descargar las que tengan más de 5.000 reseñas y una valoración media de 4 estrellas (1 es la más baja y 5 la máxima) y leer las opiniones expresadas por los clientes. El número de descargas es otro indicador fiable pues se relaciona con el nivel de satisfacción de los usuarios. Conviene seleccionar las que pasen de 10.000-50.000 descargas.

- Conviene elegir apps que se actualicen con frecuencia ya que las actualizaciones se orientan a mejorar su seguridad y funcionalidad. Conviene no descargar las que lleven más de 2-4 meses sin actualizarse.

- Como opción ética, el profesorado de EF debe priorizar el software libre y las aplicaciones gratuitas, frente al propietario de pago. Si dos apps tienen las mismas prestaciones, se debería elegir la que tenga menor coste económico para el alumnado, garantizando así la equidad y la no discriminación del alumnado con menor poder adquisitivo.

- Los centros educativos y las aulas de EF deberían ser espacios libres de publicidad, por lo que se debe asegurar que las apps no expongan a las familias, alumnado o al profesorado a mensajes, o reclamos publicitarios innecesarios.

- Cuando sea posible, hay que optar por las aplicaciones multiplataforma ya que funcionan con los sistemas operativos más usados (iOS, Android) y se comunican con los dispositivos de escritorio; lo que permitirá trabajar indistintamente desde el patio, el aula o desde casa a través de múltiples dispositivos.

- Los dispositivos móviles tienen una memoria limitada por lo que interesa elegir apps con una buena relación entre prestaciones y memoria consumida. También hay que conocer el tamaño que ocupa su descarga y el sistema operativo que requiere su instalación, para evitar saturar el hardware utilizado y garantizar su correcto funcionamiento.

Con independencia de estos criterios técnicos, se evidencia que el alumnado aprende mejor cuando está cognitivamente activo y comprometido, cuando las experiencias de aprendizaje son significativas y socialmente interactivas y cuando el aprendizaje se guía por objetivos específicos (Hirsh-Pasek et al., 2015; Papadakis \& Kalogiannakis, 2017).

\section{Usabilidad tecnológica, pedagógica y criterios éticos para evaluar la calidad de las Apps en Educación Física}

Winslow, Dickerson \& Lee (2013) indican que la elección del software adecuado es tan importante como saber utilizarlo. Para asegurar que los investigadores, el profesorado de EF y los desarrolladores de apps puedan diseñar o seleccionar aplicaciones de calidad, se necesitan marcos de referencia, con indicadores válidos, sobre la usabilidad tecnológica y la utilidad pedagógica del software.

Existen instrumentos para evaluar la calidad del software tradicional, sin embargo, esos modelos no sirven para evaluar la calidad de la tecnología móvil (Cherner, Lee, Fegely \& 
Santaniello, 2016; Papadakis \& Kalogiannakis, 2017; Zhang \& Adipat, 2005). El rápido crecimiento del mobile learning ha impulsado numerosos estudios (Aznar, Cáceres, Trujillo \& Romero, 2019; Nayebi, Desharnais, \& Abran, 2012), normas para su estandarización (p.e. ISO 9241-11, 2018) y herramientas y rúbricas que calculan la usabilidad tecnológica de los dispositivos móviles (Cherner, Dix \& Lee, 2014; Cherner et al., 2016; Papadakis, Kalogiannakis \& Zaranis, 2017). Para el profesorado, el análisis de la usabilidad tecnológica, centrado en métricas como la eficiencia, la satisfacción del usuario y la facilidad de aprendizaje, no es suficiente garantía de calidad. También se necesita valorar la usabilidad pedagógica (Turpo, 2018) de la tecnología móvil y considerar otros aspectos como la seguridad, la privacidad y la dimensión ética de su uso (p.e. Casey, Goodyear \& Armour, 2017; Eberline \& Richards, 2013; Goodyear, 2017).

La ausencia de un marco de evaluación actualizado y específico nos anima a presentar una herramienta para medir la calidad de las aplicaciones móviles de educación física CAMEF (Tabla 1). Su diseño parte de los criterios de usabilidad tecnológica y pedagógica, de las pautas éticas propuestas por la literatura de referencia y se apoya en normas y estándares internacionales (p.e. Cherner et al., 2016; Goodyear, 2017; ISO 9241-11, 2018; Nayebi, Desharnais \& Abran, 2012; Walker, 2011). El instrumento tiene 30 indicadores para valorar la calidad educativa de las apps móviles en EF, categorizadas en tres dominios: a) La

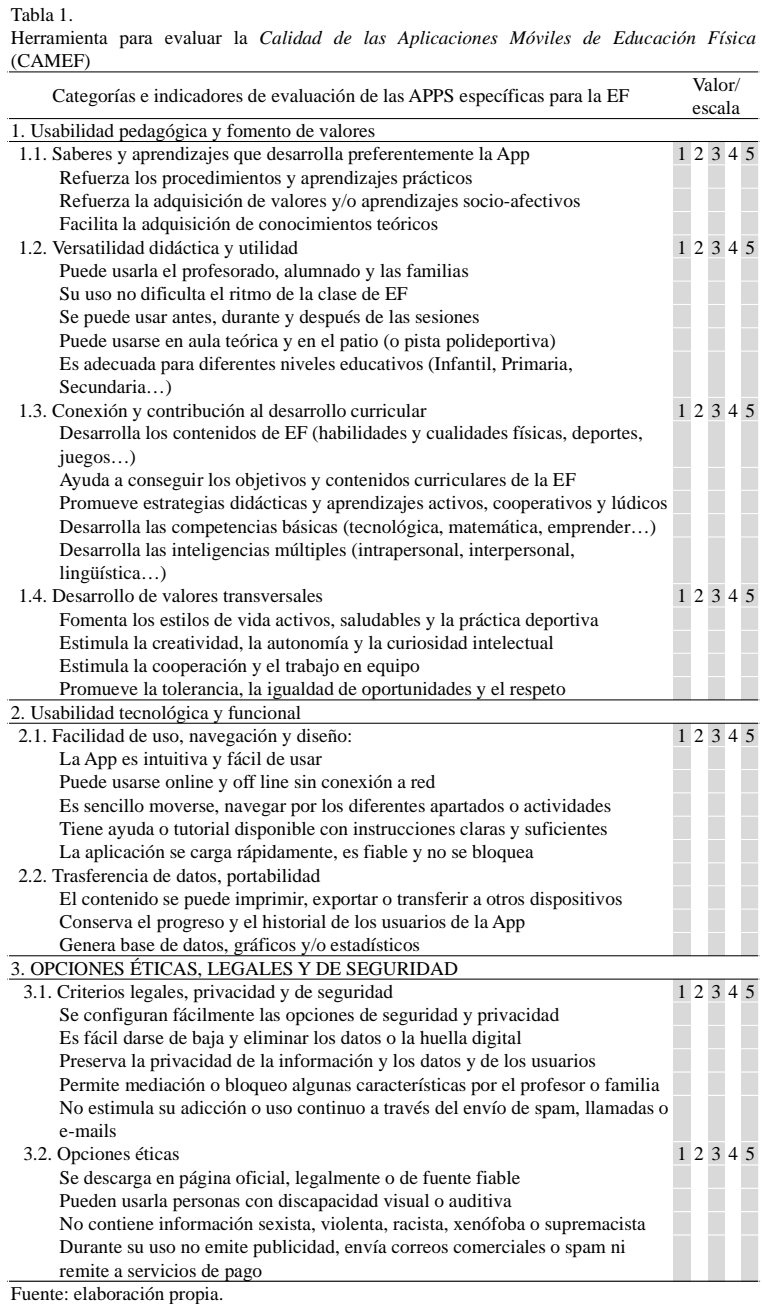

usabilidad tecnológica (17 ítems), que evalúa la facilidad de uso, la navegación o el diseño; b) La usabilidad pedagógica (8 ítems), que mide la versatilidad, la utilidad didáctica y la contribución al desarrollo curricular; c) Los criterios éticos, de seguridad y de privacidad que garantiza la aplicación (9 ítems). Se establece una escala Likert de 1 a 5 (donde: 5 = Totalmente de acuerdo; 4 = De acuerdo; 3 = Indiferente; 2 = En desacuerdo; 1 = Totalmente en desacuerdo). La valoración de la idoneidad y calidad de la app se obtiene sumando el valor de cada indicador o métrica evaluada. Cuanto más elevado es el valor resultante, mayor es la idoneidad y usabilidad tecno-pedagógica de la app evaluada. Cuando el sumatorio de los diferentes indicadores arroja valores de entre 320-245, la app es muy recomendable, entre 244-150 es recomendable y entre 149-75 no recomendable. La app es descartable cuando esas métricas de calidad y usabilidad sean inferiores a los 75 puntos.

CAMEF constituye una propuesta inicial, un instrumento que busca ofrecer a los investigadores, profesores y desarrolladores, dominios, métricas e indicadores específicos de calidad y usabilidad. Convendría validarla (o diseñar instrumentos similares) a través de estudios de campo, experimentos de laboratorio o mediciones prácticas como proponen Nayebi, Desharnais \& Abran (2012), porque el profesorado de EF necesita certificar el rigor y el valor del material curricular con el que trabaja y con el que aprende su alumnado.

\section{Dispositivos y aplicaciones móviles para la enseñanza- aprendizaje de la Educación Física}

El dinamismo de la tecnología móvil y el uso generalizado de dispositivos, especialmente teléfonos inteligentes y tabletas (Traxler, 2017), permite a los educadores físicos adoptar nuevos modelos pedagógicos (e-learning, mobile o blanded learning, flipped teaching, etc.) y mejorar la enseñanza (Jacinto, 2018). Para aprovechar su potencial didáctico, el profesorado debería conocer el hardware y el software móvil que tiene a su alcance y valorar de forma crítica sus prestaciones y limitaciones (Papadakis \& Kalogiannakis, 2017).

Respecto a los dispositivos móviles para la enseñanza de la EF, Sharples \& Beale, (2003) indican que es tan diverso que cualquier intento de clasificación y compilación puede resultar estéril. En estos momentos el profesorado de EF dispone, por señalar algunos ejemplos, de podómetros, acelerómetros, monitores de ritmo cardíaco con GPS (Geonaute o Polar), ordenadores portátiles, teléfonos inteligentes, tabletas e iPads o videocámaras digitales. También de consolas de videojuegos activos (Play Statation, Xbox), videocámaras de acción tipo GoPro, proyectores sin cables (p.e. LG PH450UG LED) o simuladores de realidad virtual o realidad aumentada. Las prestaciones de los dispositivos no dejan de crecer y mejorar. Por ejemplo, el iPad Pro o el iPhone 6 incorporan una cámara de vídeo de alta velocidad (240fps), con 3-D, acelerómetros y giroscopios con los que realizar un análisis exhaustivo de la motricidad. La tecnología usable (Wearable Technology) y sus sensores y complementos robots deportivos, Smart card, Smart watch-, los drones y los accesorios informáticos instalados en ropa y zapatillas también están llamados a operar una gran evolución en las 
aulas de EF y en el ámbito deportivo. Para McNab, James \& Rowlands (2011), el desarrollo y refinamiento continuo de los dispositivos sigue una tendencia caracterizada por la disminución del precio y el tamaño de los dispositivos y el aumento exponencial de sus prestaciones (metrificación, precisión, etc.). La escuela no debería ser ajena a esta realidad, aunque la resistencia al cambio, la falta de formación de una parte del profesorado o el coste económico del hardware, están dificultando su incorporación educativa (Aubusson, Schuck \& Burden, 2016; Díaz, 2018).

En relación a las aplicaciones móviles para la EF, se vienen documentando múltiples usos y experiencias de integración didáctica (p.e. Cummiskey, 2013; Lavay et al., 2015; Phillips, Rodenbeck \& Clegg, 2014; Rosenthal \& Eliason, 2015; Yankov \& Maion, 2014; Yu, Kulinna \& Lorenz, 2018). Estas fuentes y consultas realizadas entre diciembre de 2017 y mayo de 2018, en la Google Play Store y la App Store de iOS, relacionadas con diversas categorías vinculadas a la EF (p.e. juegos, deportes o salud), muestran la gran variedad de apps que existen para su enseñanza. Aplicaciones para gestionar la comunicación, el correo electrónico y las aulas virtuales (Moodle, Edmodo). Para realizar vídeo-conferencias (Hangouts, Skype, Face Time) o para conectar escuela y familias y compartir recursos formativos (Whatsapp, Gmail, Remind, DropBox, Drive, Doodle). Otras sirven para editar, crear o enviar material curricular con el que dinamizar pedagogías emergentes -como la flipped classroom- o reforzar el aprendizaje de conceptos teóricos (p.e. Lensoo Create, Screen Chomp, Educareations, ShowMe o Explain everything). Las apps de foto y vídeo se usan para tomar imágenes, escanear y archivar justificantes, partes médicos o calificaciones (Scannable, Cam Scanner) o grabar montajes, coreografías o competiciones, dando feed-back inmediato al alumnado (Technique). El micrófono asociado al sistema de reconocimiento de voz y apps como Dragon Dictation o Voice Assistant, transcribe la voz a texto y agiliza la redacción de informes y entrevistas. Con Class Dojo, IDoceo, Additio o Time Motion, se simplifican las tareas de gestión y control del aula. Tareas habituales y sencillas, como controlar el tiempo de ejecución-recuperación (Seconds, Tabata), organizar grupos (Team Shake, Team Make, Teams iDoceo) o puntuar los juegos deportivos (Giant Timer, Circle Timer, Scoreboard) también se simplifican con apps.

La evaluación y retroalimentación de la enseñanza-aprendizaje, el elemento más complejo del acto didáctico (Díaz, 2018), puede mejorarse con Live Video Delay, Coach's Eye, Video Delay BAM o Sportstec GB. Otras apps añaden rigor en la valoración del rendimiento físico-deportivo con acelerómetros y monitores de ritmo cardíaco (Icare Monitor, Heart Bits, My Heart Rate, Polar Club), podómetros (Podometer, Moves) o ayudan a personalizar las cargas de trabajo (XTrain C-Fit, Sworkit Kids, Personal Fitness Coach). También miden el rendimiento atlético (Bleep Test, Test Cooper) y cualidades físicas como la velocidad (MySprint) o la potencia de piernas (MyJump). Trabajos de Balsalobre-Fernández, Glaister \& Lockey (2015) o Williams, Caserta \& Haines (2013), confirman que cada vez más entrenadores, profesores o fisioterapeutas usan apps, validadas científicamente, para realizar análisis posturales (Posture Aware, Mugikon), evaluar la marcha (RunMatic) o la inclina- ción angular (TiltMeter). Las apps no dejan de crecer y, como señala Díaz (2015), en estos momentos se puede encontrar una aplicación para cada función didáctica.

\section{Marcos y estrategias para integrar el mobile learning en la educación física}

Para Carr (2011) la tecnologización de la escuela es una realidad que hay que aceptar y asumir porque es imparable. La opción del profesorado no es plantear TIC sí o TIC no. La opción realista consiste en decidir cómo y para qué introducir tabletas o teléfonos inteligentes en las aulas de EF (Koekoek \& Van-Hilvoorde, 2018). En este contexto, la formación digital del profesorado adquiere notable relevancia, máxime cuando asistimos a un cambio de discurso formativo que supera la inicial hegemonía tecno-céntrica y se orienta a modelos que priman lo pedagógico sobre lo tecnológico (Casey, Goodyear \& Armour, 2016; Eberline \& Richards, 2013). Así lo reflejan los actuales estándares para la formación digital del profesorado y la aparición de numerosos marcos de integración digital como el SMAR (Substitution, Augmentation, Modification, Redefinition) de Puentedura, o el TPCK (Technological, Pedagogical and Content Knowledge) de Mishra \& Koehler (2006). Marcos que sirven de referencia para la aplicar modelos tecno-pedagógicos como la gamificación, el blanded learning o de técnicas didácticas como el flipped classroom o el aprendizaje servicio (ApS) entre otros.

Esta redefinición y sinergia tecno-pedagógica, sumada al empuje de la tecnología móvil, está posibilitando la creación de modelos didácticos específicos para integrar el mobile learning en la escuela. Algunos modelos de referencia se centran en los procesos de enseñanza-aprendizaje, como el de Dick, Carey \& Carey (2005) o en los entornos de formación docente, como ASSURE (Analyse, State objetives, Select, Utilize materials, Require learner response, Evaluate) de Smaldino, Russell, Heinich \& Molenda (2005). Otros resaltan la dimensión ética, como el M-COPE de Dennen \& Hao (2014), adaptado a la educación física por Wyant \& Baek (2018). La mayoría coinciden en señalar como factores relevantes: el rol del alumnado, las características de los dispositivos, las condiciones del contexto de aprendizaje y las directrices o modelos didáctico-pedagógicos que apoyan el aprendizaje móvil o lo asocian al constructivismo, a las pedagogías activas y al aprendizaje permanente (Peng, Su, Chou \& Tsai, 2009).

También existen modelos de aprendizaje móvil centrados en la posesión de medios tecnológicos. Entre ellos el más exitoso es el Bring Your Own Device (BYOD), donde profesorado y alumnado llevan sus dispositivos al centro educativo para enseñar y aprender (CastilloManzano, Castro-Nuño, López-Valpuesta, Sanz-Díaz \& Yñiguez, 2017; Song, 2014). Habría que investigar si el BYOD, que incentiva el aprendizaje y la participación del alumnado, puede mejorar la enseñanza de la EF, máxime considerando que el $98 \%$ de los jóvenes de entre 10-14 tiene un smartphone en propiedad (International Telecommunication Union, 2016) que podría utilizar en las clases.

Para introducir el mobile learning en EF, estos modelos, marcos y estándares de referencia son necesarios, pero no 
suficientes (Acaso, 2013; Casey, Goodyear \& Armour, 2016; Rosenthal \& Eliason, 2015); también se deben concretar proyectos de inmersión digital ad hoc, progresivos y consensuados con toda la comunidad educativa (Díaz, 2018). Algunos criterios y líneas estratégicas que sugiere la literatura (Cummiskey, 2013; Dennen \& Hao, 2014; Díaz, 2018; Loureiro, 2012; Yu, Kulinna \& Lorenz, 2018) para lograrlo son:

- Partir de un análisis y un diagnóstico previo de los recursos y servicios necesarios, del profesorado colaborador y de los costes económicos, estableciendo los plazos de implementación progresiva.

- Diseñar una planificación a corto, medio y largo plazo con objetivos de aprendizaje explícitos y realistas y evaluarlos de forma continua.

- Determinar cómo será el mantenimiento de servicios y hardware: la carga de los dispositivos, la prevención ante virus, roturas y deterioros o el tiempo de obsolescencia. Contemplar otras cuestiones técnicas como el servicio wifi a contratar, sus costes, su potencia y mantenimiento.

- Especificar qué modelo de dispositivo y de apps se van a necesitar y quién costeará su adquisición, mantenimiento y reposición.

- Introducir el plan de inmersión digital en el Reglamento de Régimen Interno del centro estableciendo cómo, cuándo y para qué se usarán los dispositivos, en clase y en horarios no lectivos, asesorando a las familias para su uso extraescolar

- Decidir qué modelo pedagógico respaldará su integración: BYOD, SMAR, TPACK, etc., formando al profesorado, alumnado y al resto de la comunidad educativa de manera transversal y permanente.

- El plan de integración digital, además de las finalidades didácticas, debe considerar otras derivas de la CD: su dimensión social, ética y crítica y aspectos nucleares como la seguridad, la privacidad y la protección de datos (Aubusson, Schuck \& Burden, 2016; Traxler, 2016).

- El proyecto se debería acompañarse de un plan de formación de mediadores digitales que involucre especialmente al alumnado, para evitar malas prácticas y abusos (sobreexposición digital, tecnoadicciones o ciberacoso) y prevenir brechas digitales por motivos económicos, de género o intergeneracionales. Este hipotético plan se tendría que evaluar y realimentar de forma permanente.

Aunque el profesorado puede tomar como referencia los marcos y estrategias referidos, convendría diseñar modelos ad hoc para la integración del mobile learning en la EF, porque actualmente no existen. Dichos modelos deben mejorar las interacciones entre el profesorado, el alumnado y el uso de la tecnología, con la finalidad de enriquecer las experiencias de aprendizaje basadas en, para y sobre la acción y en la reflexión crítica (Koekoek \& Van-Hilvoorde, 2018; Schön, 1987).

\section{Creencias, optimismo y visión distópica sobre los dispo- sitivos y las aplicaciones móviles}

Los dispositivos móviles poseen utilidades innegables: multifuncionalidad, portabilidad, compatibilidad y menor coste, pero no todo son ventajas (Lavay et al., 2015). Table- tas y smartphones son excelentes herramientas para la comunicación, el acceso y la reproducción de contenidos, sin embargo, son incómodos para trabajos de gestión y de creación de contenidos y tienen menor capacidad de almacenamiento o utilidad offline. Para Aubusson, Schuck \& Burden, (2016) o Traxler (2016), su uso está condicionado por determinadas creencias y recelos del profesorado sobre la vulnerabilidad de las redes, la privacidad de los datos personales -académicos, médicos o disciplinares- y por actitudes prejuiciosas sobre la validez y la fiabilidad de las apps. Estas creencias explican parcialmente por qué los centros educativos siguen vetando su uso (Garmendia-Larrañaga et al., 2016; Loureiro, 2012).

Los datos del Informe Ditrendia (2017), acreditan la existencia de más dispositivos móviles (7,9 mil millones) que personas en el planeta y que el mercado de los accesorios digitales corporales es uno de los más desarrollados. Esta penetración de wearables que interactúan continuamente con el usuario, se ve con optimismo y con recelo (Cummiskey, 2013; Wyant \& Baek 2018). La visión optimista les atribuye el potencial de promover la actividad física saludable las veinticuatro horas al día, siete días a la semana, pero otras evidencias son más escépticas (p.e. Sandercock, Alibrahim \& Bellamy, 2016) indicando que los dispositivos móviles, especialmente smartphones y tabletas, pueden causar aislamiento social y procastinación o promover el sedentarismo y el empobrecimiento motriz entre la población escolar.

La situación es paradójica. Por una parte, los dispositivos permiten a las personas monitorear y documentar sistemáticamente muchos aspectos de su vida: estados de ánimo, patrones de sueño, rendimiento físico, ingesta calórica, etc., otorgándoles un autoconocimiento y empoderamiento profundo (Wolf, 2010). Por otra, se rechaza ese discurso utópico de la autonomía en la mejora de la salud y se denuncia la cuantificación de los cuerpos y los riesgos del auto-monitoreo permanente (p.e. Lomborg \& Frandsen, 2015; Lupton, 2013; 2014a). En todo caso, la avalancha de datos que ofrecen los dispositivos móviles no debe restar protagonismo a los profesionales de la de EF o de la salud, como teme Lupton (2014b), ya que esos deslumbrantes dispositivos -con sus miles de datos y bits- necesitan supervisión profesional. Entrenarse, alimentarse o seguir una dieta de adelgazamiento, siguiendo exclusivamente las indicaciones de una app, puede ser tan aventurado como automedicarse sin control facultativo.

Respecto a las dudas de algunos profesionales de la EF y la salud sobre la precisión y la fiabilidad de las apps para medir el rendimiento deportivo o sobre su valor para fomentar la actividad física entre los usuarios, existe literatura que refuta esas creencias. Millington (2014) desmiente que las apps de salud y fitness, promuevan el aislamiento o el individualismo destacando su creciente dimensión social. Así lo evidencian aplicaciones como MyFitnessPal, Zombies, Run!, Runastic, Wikiloc y otras que crean redes sociales deportivas que conectan a los usuarios para que practiquen deporte (p.e. Yes we play, Timpik, Sport Meeting o Fubles). Trabajos de O’Loughlin-J., Sabiston, Dugas \& O’Loughlin-E (2015) con exergamers, o de Pulido, Sánchez, Sánchez-Miguel, González \& García (2016), constatan la satisfacción de profesorado y alumnado y el incremento de niveles de actividad 
física en escolares cuando usan apps.

Sobre la escasa fiabilidad de las aplicaciones móviles, especialmente las gratuitas, para determinar indicadores médicos, de fitness o para analizar el rendimiento motriz, existen evidencias que equiparan la precisión de las apps con los medios tradicionales de laboratorio. Así, BalsalobreFernández, Glaister \& Lockey (2015) verifican el rigor de My Jump para medir la potencia del salto vertical, comparándola con plataformas de fuerza tradicionales; Pourahmadi, Taghipour-Jannati, Mohseni-Bandpei, Takamjani y Rajabzadeh (2016) prueban la validez y confiabilidad de MySprint, midiendo los resultados de sprint comparándolos con métodos de campo existentes; o Romero-Franco, et al., (2016) certifican la validez de TiltMeter para evaluar la flexoelasticidad de la columna lumbar y otras propiedades relacionadas con la inclinación angular (Williams, Caserta \& Haines, 2013). Repositorios médicos como PubMed, HealthGate o BioMedNet, muestran el rigor y utilidad físicomédica de numerosas apps; aunque obviamente no todas las que se encuentran en el mercado cumplen con los estándares de fiabilidad y validez deseados.

\section{Seguridad, privacidad y protección de datos: el debate pendiente}

La escuela nunca ha generado y usado tanta información, personal y académica, como en la actualidad. Sin embargo, esta producción masiva de información no se acompaña de una mayor capacidad para garantizar su privacidad. En los escenarios digitales la seguridad $100 \%$ no existe, por ello al introducir la tecnología móvil a las aulas las hacemos más vulnerables (Garmendia-Larrañaga et al., 2016). La comunidad educativa deber proteger la información relacionada con las prácticas pedagógicas y gestionarla de forma segura, legal y ética (Goodyear, 2017). Pero ¿sabe el profesorado de EF qué permisos otorga y qué información cede cuando descarga una app tipo Runtastic, Seven, Class Dojo, RunKeeper o Endomondo? Hablamos de información y datos personales sensibles como la localización, los hábitos de vida o el estado de salud. Estas reflexiones, que eran innecesarias hasta la fecha, ahora son imprescindibles, máxime cuando investigaciones sobre los dispositivos de salud y fitness constatan la existencia de brechas de seguridad (p.e. Huckvale, Prieto, Tilney, Benghozi \& Car, 2015). Sin compartir las actitudes tecno-escépticas de algunos profesionales de la EF destacados en la literatura (Ferreres, 2011; Díaz, 2015), o los escenarios distópicos que plantean la integración de las TIC (Gard, 2014; Lupton, 2014a; Williamson, 2015), debería asumirse que un entorno académico digitalizado representa tantos desafíos como oportunidades (Carr, 2011).

Diversas instituciones nacionales e internacionales ya están trabajando para sensibilizar sobre la importancia de la seguridad de personas, dispositivos y datos. En mayo de 2018, entró en vigor el Reglamento General de Protección de Datos Personales (UE 2016/679) de la Unión Europea, legislando sobre los derechos digitales de los menores, la protección y la privacidad de datos masivos. Entidades como la Agencia Española de Protección de Datos (p.e. http:// www.tudecideseninternet.es/agpd1/) o la Oficina para la Seguridad del Internauta (OSI) (https://www.osi.es/es), elabo- ran continuos informes, recursos y material didáctico dirigido a la comunidad educativa. También aumentan los estudios sobre el impacto de las tecnologías en la protección de los datos en menores de edad (p.e. Garmendia-Larrañaga, et ál., 2016; Golob, 2015; Jaroszek, 2015). Algunas líneas que sugieren para incrementar la seguridad son: configurar la privacidad de los dispositivos ante posible pérdida o sustracción, activar una contraseña de desbloqueo, ser cauteloso al conectarse a wifi públicas o a través del bluetooth y no anular las restricciones del fabricante sobre la seguridad de los dispositivos. También conocer qué permisos se autorizan al descargar una aplicación, mantener el navegador actualizado, borrar el historial de navegación no utilizado, eliminar con frecuencia las cookies o cerrar la sesión cuando se trabaja en dispositivos compartidos que piden autenticar el usuario y contraseña. Para que los equipos no sean vulnerables conviene instalar complementos de protección del hardware pues los dispositivos móviles (smartphones y tabletas), necesitan la misma protección que los equipos de sobremesa. El software antivirus es necesario con independencia de su sistema operativo. Para iOS (iPhone iPad) se puede instalar: Lockout Mobile, Norton Internet Security o Avira Mobile Security; para proteger dispositivos Android: ¡Bitdefender, Avast! O AVG Antivirus, entre otros. Descargar apps de canales de distribución no oficiales aumenta el riesgo de dañar los aparatos con virus y malware.

Otra reflexión en torno a la seguridad, se refiere a la portabilidad y el uso de dispositivos móviles usados en entornos abiertos y activos como las clases de EF. En dicho contexto, advierten Lavay et al., (2015), la utilización de tabletas o smartphones incrementan el riesgo de rotura por caídas y golpes. Igualmente pueden dañarse por la acción de agentes externos como el agua o las altas temperaturas, por lo que conviene protegerlos con accesorios como fundas impermeabilizantes, carcasas anti-golpeos, bolsas con correas hombro-tronco o agarraderas de mano. Estos elementos de seguridad no deben restar funcionalidad a los dispositivos, para lo cual hay que mantener despejada la cámara delantera-trasera, la entrada de audio o el micrófono. Tampoco se deben dificultar o limitar las posibilidades de acción del profesorado (Díaz, 2018). Es aconsejable contratar una póliza de seguro que cubra eventuales desperfectos de los dispositivos.

\section{Los retos del aprendizaje móvil en la docencia y la inves- tigación de la EF}

La institución educativa y su profesorado están convencidos de que el futuro está vinculado a procesos de inmersión digital, voluntarios o involuntarios, pero en todo caso irreversibles. Mientras crece el discurso de la conveniencia y las ventajas de la integración los dispositivos móviles en la escuela, lo cierto es que todavía no hemos sabido cómo integrarlas (Traxler, 2017; Wyant \& Baek, 2018). Sabemos para qué, pero el reto es concretar cómo llevar a cabo esa integración digital agregando bases académicas al aprendizaje móvil. Con independencia de las propuestas realizadas en apartados anteriores, se sugieren algunas líneas de debate, investigación e innovación en torno al mobile learning y las apps en la EF. 
En primer lugar, tecnólogos y pedagogos han de trabajar en equipo para resolver algunas limitaciones técnicas de los dispositivos móviles, aumentando su capacidad de almacenamiento, su utilidad offline, la conexión mediante voz o mejorando la usabilidad de teclados y pantallas, hoy incomodas y pequeñas. En esta línea, Yu, Kulinna \& Lorenz (2018) o Aznar, Cáceres, Trujillo \& Romero (2019), piden al profesorado una mayor proactividad hacia las tecnologías emergente, más implicación en el desarrollo de herramientas educativas, como resuelven Ariza, Fernández-Jiménez, Muñoz-Calle, Sierra \& Mora (2019), y mayor cooperación entre escuelas, empresas, universidades y organizaciones profesionales.

Como proponen los diferentes marcos que desarrollan la CD docente (p.e. INTEF, 2017), profesorado y alumnado deben convertirse en «prosumidores» (Toffler, 1980) desarrollando competencias para consumir y producir cultura y conocimiento digital, diseñar entornos personales de aprendizaje (EPA), curar contenidos o producir materiales curriculares ad hoc. En esta línea, algunos profesionales de EF ya están creando apps para sus clases (p.e. Educación física 3.0, de Santi Querol), aprovechando la existencia de herramientas de programación que no requieren de conocimientos tecnológicos avanzados como Code.org App Lab (https:// code.org/educate/applab), Thunkable (https:// thunkable.com), o la popular AppInventor (http:// appinventor.mit.edu) que desarrollan el MIT (Massachusetts Institute of Technology) y Google, con la que alumnado y profesorado ya han diseñado más de 20 millones de apps.

Es necesario mejorar los procesos didácticos estudiando cómo transformar los dispositivos móviles o «dispositivos frontera» (Pimmer \& Groehbiel, 2011), próximos al aprendizaje formal-informal, público-privado, usados en el hogar y en la escuela, en dispositivos bisagra capaces de articular procedimientos de enseñanza-aprendizaje, que mejoren las interacciones entre profesorado y el alumnado y contribuyan a la reflexión en la acción en la EF.

Como proponen Lupton (2014a) o Goodyear (2017) conviene hacer una profunda reflexión crítica, social y ética del uso de los dispositivos móviles y las redes sociales para disipar la inquietud que están generando centrando la reflexión en torno a cuatro principios: el consentimiento informado, el anonimato, la protección contra riesgos y daños y los aspectos legales del aprendizaje móvil. Existen otros dilemas éticos que deberían ser abordados: la cuestionable participación de los usuarios y la trazabilidad de individuos y datos o el futuro impacto de la inteligencia artificial en los procesos de enseñanza-aprendizaje, como está sugiriendo la Unión Europea (Tuomi, 2018).

Es posible que el profesorado de EF, fascinado por las TIC, haya olvidado ponerles límites (Garmendia-Larrañaga et al., 2016), por lo que procede una perspectiva investigadora que aporte ideas para prevenir el ciberacoso, las adicciones, el aislamiento social o el uso inadecuado de redes sociales educativas, tan unidas a los dispositivos móviles. Un mayor uso de las TIC implica más riesgos y mayor vulnerabilidad de las redes, los dispositivos y los datos generados por la escuela; máxime cuando los dispositivos se usan en espacios abiertos -patios, pista polideportiva- donde la conectividad es necesariamente inalámbrica. Se debe garantizar la seguri- dad, la privacidad o la identidad personal y académica dentro y fuera de los recintos escolares pues la frontera escolarextraescolar se diluye.

Conviene abordar la relación singular entre las TIC y un contexto de práctica tan específico como el de la EF. ¿Cómo integrar los dispositivos móviles sin hacer más sedentarias las prácticas, ni disminuir el compromiso motor del alumnado? Si tabletas, smartphones y consolas pueden fomentan el sedentarismo, ¿Cómo transformarlas en promotoras de estilos de vida activos y saludables? También habría que determinar las características de la formación que necesita el profesorado de EF para usar tecnologías específicas en contextos singulares, como son los aprendizajes en acción.

La naturaleza expansiva y dinámica de las tecnologías móviles obligará a una constante redefinición porque plantea continuos retos: repensar constantemente el concepto de CD docente añadiendo a su dimensión tecnológica derivas sociales, éticas o legales, rediseñar la formación inicial y permanente del profesorado, intensificar el esfuerzo investigador sobre cómo puede el mobile learning resolver problemas reales. Deben plantearse más trabajos de campo cualitativos y etnográficos, pues no solo interesan cifras y datos descriptivos, también se deben conocer los argumentos, los intereses y las necesidades del profesorado y el alumnado frente a la cultura digital.

\section{Conclusiones}

En este trabajo se han analizado las posibilidades y limitaciones del aprendizaje móvil en la educación física mostrando, como indica la literatura, que estamos ante un campo relativamente inmaduro con más preguntas que respuestas. Como indica Traxler (2017), la naturaleza cambiante de las TIC, hace que estos interrogantes surjan y cambien constantemente. En este contexto, los educadores físicos y los investigadores deben esforzarse para determinar cómo y para qué integrar las TIC en la profesión.

La vinculación entre TIC y educación jamás ha sido tan evidente. Internet y especialmente las tecnologías móviles, sociales y ubicuas, han llegado para cambiarlo todo (Carr, 2011) y condicionar las formas tradicionales de enseñar, de aprender o de investigar. Esta realidad interpela a redefinir la relación docente-discente pues trabajamos con un alumnado que posee, conoce y usa de forma habitual dispositivos digitales y redes sociales.

Rich \& Miah (2016) recuerdan que los dispositivos y las apps móviles solo son mecanismos intermediarios. Es el profesorado el que debe conocer su usabilidad tecnológica y pedagógica, establecer y seguir marcos y estándares digitales de referencia y hacer una buena selección a partir del conocimiento de sus las ventajas y limitaciones. Los proyectos de inmersión digital deben orientarse hacia la mejora de la docencia, pero también a garantizar la privacidad y la seguridad de los dispositivos, los datos y los usuarios. Para conseguirlo, la formación en CD del profesorado, además de capacitar para el uso instrumental y pedagógico de las TIC, debería estimular su utilización crítica y concienciar sobre sus implicaciones sociales, éticas y legales.

Es conveniente que los profesionales de la educación física y deportiva revisen sus creencias y sus actitudes, las 
utópicas y las distópicas, sobre las TIC y sobre su valor didáctico, porque en ocasiones corresponden más a estereotipos que a ópticas realistas. La innovación y la investigación son primordiales para aprovechar el inmenso potencial de mejora y trasformación educativa que tiene la tecnología móvil en la EF. El creciente debate social, educativo y disciplinar sobre estas tecnologías sugiere que estamos al inicio de un camino que debería explorarse de forma colectiva y serena.

\section{Referencias}

Ariza, T., Fernández-Jiménez, F.J., Muñoz-Calle, J., Sierra, A.J., \& Mora, M. (2019). Arquitectura software para la prescripción de ejercicio físico personalizado. Revista Internacional de Medicina y Ciencias de la Actividad Física y el Deporte, 19(73) 137-150. doi: http://doi.org/ 10.15366/rimcafd2019.73.010

Aubusson, P., Schuck, S., \& Burden, K. (2016) Mobile learning for teacher professional learning: benefits, obstacles and issues, ALT-J, 17(3), 233-247. doi:10.1080/ 09687760903247641

Aznar, I., Cáceres, M.P., Trujillo, J.M., \& Romero, J.M. (2019). Impacto de las apps móviles en la actividad física: un meta-análisis. RETOS: nuevas tendencias en educación física, deporte y recreación, (36), 52-57.

Balsalobre-Fernandez, C., Glaister, M., \& Lockey, R.A. (2015). The validity and reliability of an iPhone app for measuring vertical jump performance. Journal of Sports Sciences, 33,1574-1579. doi:10.1080/02640414.2014.996184

Can, S. (2015). Investigation of pre-service physical education teachers' attitudes towards computer technologies (case of turkey). Educational Research and Reviews, 10(4), 485-490. doi:http://dx.doi.org/10.5897/ERR2014.1938

Carr, N. (2011). Superficiales. ¿Qué está haciendo internet con nuestras mentes? Madrid: Taurus.

Casey,A.(Ed.), Goodyear, V.A.(Ed.), Armour, K. (Ed.). (2017). Digital Technologies and Learning in Physical Education. London: Routledge.

Casey,A., Goodyear, V.A., \& Armour, K. M. (2016). Rethinking the relationship between pedagogy, technology and learning in health and physical education. Sport, education and society, 22(2), 288-304. doi:10.1080/ 13573322.2016.1226792

Castillo-Manzano, J., Castro-Nuño, M., López-Valpuesta, L., Sanz-Díaz, M.T., Yñiguez, R. (2017) To take or not to take the laptop or tablet to classes, that is the question. Computers in Human Behavior, (68), 326-333. doi:org/10.1016/j.chb.2016.11.017

Cherner, T., Dix, J., \& Lee, C. (2014). Cleaning up that mess: A framework for classifying educational apps. Contemporary Issues in Technology and Teacher Education, 14(2), 158-193. En: https:// www.learntechlib.org/p/129859/

Cherner, T., Lee, C-Y., Fegely, A., \& Santaniello, L. (2016). A detailed rubric for assessing the quality of teacher resource apps. Journal of Information Technology Education: Innovations in Practice, 15, 117-143. De: http:/ /www.informingscience.org/Publications/3527

Crawford, S., Fitzpatrick P. (2015) Use of mobile digital technology and iPod Touch in physical education, In: Zhang Y. (eds). Handbook of Mobile Teaching and Learning. Wollongong, (499-508), NSW, Australia. WEMOSOFT. En: https://ink.springer.com/content/pdf/ 10.1007\%2F978-3-642-54146-9.pdf

Cummiskey, M. (2013). There's an App for That Smartphone Use in Health and Physical Education, Journal of Physical Education, Recreation \& Dance, 82(8), 2430. doi:10.1080/07303084.2011.10598672

Dennen, V.P., \& Hao, S. (2014) Intentionally mobile pedagogy: the M-COPE framework for mobile learning in higher education. Technology, Pedagogy and Education, 23(3), 397-419. doi:10.1080/ 1475939X.2014.943278

Díaz, J. (2015). La competencia digital del profesorado de educación física en educación primaria: Estudio sobre el nivel de conocimiento, la actitud, el uso pedagógico y el interés por las TIC. (Tesis Doctoral inédita). Universitat de València. València.

Díaz, J. (2018). Mejorar el aprendizaje en acción integrando mobile learning en la educación física. Tándem: Didáctica de la educación física, (60), 69-73.

Dick, W., Carey, L., \& Carey, J. O. (2005). The systematic design of instruction (6th ed.). Upper Saddle River, NY: Pearson.

Dickerson, J., Winslow, J., \& Lee, C. Y. (2013). Teacher training and technology: Current uses and future trends. En Wang, V. (Ed.). Handbook of Research on Technologies for Improving the 21st Century Workforce: Tools for Lifelong Learning, 243-256. USA: IGI Global. Florida Atlantic University.

Ditrendia (2017). Informe Ditrendia: Mobile en España y en el mundo. En: https://www.amic.media/media/files/ file_352_1289.pdf

Eberline, A.D., \& K. Richards, A. R. (2013). Teaching with Technology in Physical Education, Strategies, 26(6) 3839. doi:10.1080/08924562.2013.839522

El-Hussein, M. O. M., \& Cronje, J. C. (2010). Defining mobile learning in the higher education landscape. Educational Technology \& Society, 13(3), 12-21.

European Union. (2016). Regulation (EU) 2016/679 of the European Parliament and of the Council on the protection of natural persons with regard to the processing of personal data and the free movement of such data and repealing Directive 95/46 / EC (General data protection regulation. Official Journal of the European Union, 4. En: https://publications.europa.eu/en/publication-detail/-/ publication/3e485e15-11bd-11e6-ba9a-01aa75ed71a1/ language-en

Feixa, C., Fernández-Planells, A., \& Figueras-Maz, M. (2016). Generación Hashtag. Los movimientos juveniles en la era de la web social. Revista Latinoamericana de Ciencias Sociales, Niñez y Juventud, 14(1), 107-120.

Ferreres, C. (2011). La integración de las tecnologías de la información y de la comunicación en el área de la educación física de secundaria: Análisis sobre el uso, nivel de conocimientos y actitudes hacia las TIC y de sus posibles aplicaciones educativas. (Tesis Doctoral). Universitat Rovira i Virgili. En: TDX/TDR database, en: http://www.tesisenred.net/handle/10803/52837 
Gard, M. (2014). eHPE: A history of the future. Sport Education and Society, 219(6), 827-845.

Gardner, H., \& Davis, K. (2014). La generación app: cómo los jóvenes gestionan su identidad, su privacidad y su imaginación en el mundo digital (12). Barcelona: Paidós(GBS).

Garmendia-Larrañaga, M.; Jiménez-Iglesias, E.; Casado-del-Río, M.A.; Mascheroni, G. (2016). Net children go mobile: Riesgos y oportunidades en internet y el uso de dispositivos móviles entremenores españoles(2010-2015). Madrid:Red.es; Universidad del País Vasco. https:/addi.ehu.es/handle/10810/ 21546

Gibbone, A., Rukavina, P., \& Silverman, S. (2010). Technology integration in secondary physical education: teachers' attitudes and practice. Journal of Educational Technology Development and Exchange, 3(1), 27-42.

Golob, B. (2015). How safe are safe harbors? The difficulties of self-regulatory children's online privacy protection act programs. International journal of communication, (9), 34693476. En:http://ijoc.org/index.php/ijoc/article/view/3327

Goodyear, V. A. (2017). Social media, apps and wearable technologies: navigating ethical dilemmas and procedures. Qualitative research in sport, exercise and health, 9(3), 285-302 doi: 10.1080/2159676X.2017.1303790

Hirsh-Pasek, K., Zosh, J. M., Golinkoff, R. M., Gray, J. H., Robb, M. B., \& Kaufman, J. (2015). Putting education in «educational» apps: Lessons from the science of learning. Psychological Science in the Public Interest, 16(1), 3-34. doi.org/10.1177/1529100615569721

Huckvale, K., Prieto, J. T., Tilney, M., Benghozi, P-J., \& Car, J. (2015). Unaddressed privacy risks in accredited health and wellness apps: A cross-sectional systematic assessment. BMCMedicine, 13(1), 214. doi: 10.1186/s12916015-0444-y

International Telecommunication Union, ITU.(2016). Measuring the Information Society Report. En: https://www.itu.int/pub/ D-IND-ICTOI-2016

ISO International Standard, ISO 9241-11 (2018). Ergonomic requirements for office work with visual display terminals (VDTs.), Part 11: Guidance on usability, En: https:// www.iso.org/obp/ui/\#iso:std:iso:9241:-11:ed-2:v1

Jacinto, J.J. (2018). Aplicações das TIC no ensino da educação física. RETOS. Nuevas Tendencias en Educación Física, Deportey Recreación, (34), 371-376.

Jaroszek, A. (2015). Online behavioural advertising and the protection of children's personal data on the Internet. Wroclaw review of law, administration \& economics, 4(2), 56-65. doi:10.1515/wrlae-2015-0015

Koekoek, J. (Ed.), Van-Hilvoorde, I. (Ed.). (2018). Digital Technology in Physical Education. London: Routledge.

Kretschmann, R. (2017, October). Significance of Technology Integration in Higher Education Classes: How do Kinesiology/Sport Science Students Rate Technology use in Gym-Based and Classroom-Based Courses? European Conference on e-Learning, 281-288. Academic Conferences International Limited. En: https://search.proquest.com/ docview/1968935394?accountid=14777

Lavay, B., Sakai, J., Ortiz, C., \& Roth, K. (2015). Tablettechnology to monitor physical education IEP goals and benchmarks. Journal of Physical Education, Recreation \& Dance, 86(6), 16-23.doi:10.1080/07303084.2015.1053633
Lomborg, S., \& Frandsen, K. (2015). Self-tracking as communication, Information, Communication \& Society, 19(7), 1015-1027.doi:10.1080/1369118X.2015.1067710

Loureiro, M. (30 de noviembre de 2012). Sobrelaprohibición del uso de teléfonos móviles en escuelas e institutos. Educa con TIC. En: http://www.educacontic.es/blog/sobre-laprohibicion-del-uso-de-telefonos-moviles-en-escuelas-e-institutos

Lupton, D. (2013). Quantifying the body: Monitoring and measuring health in the age of mHealth technologies. Critical Public Health,23,393-403.doi:10.1080/09581596.2013.794931

Lupton, D. (2014a). Critical perspectives on digital health technologies. Sociology Compass, 8(12), 1344-1359. doi:org/ 10.1111/soc4.12226

Lupton, D. (2014b). Data assemblages, sentient schools and digitized health and physical education (response to Gard). Sport, Education and Society, 20(1), 122-132. doi:org/ 10.1080/13573322.2014.962496

McNab, T., James, D. A., \& Rowlands, D. (2011). iPhone sensor platforms: Applications to sports monitoring. Procedia Engineering, 13, 507-512.

Millington, B. (2014). Smartphone Apps and the Mobile Privatization of Health and Fitness. Critical Studies in Media Communication, (31),5, 479-493, doi: 10.1080/ 15295036.2014.973429

Mishra, P., \& Koehler, M. J. (2006). Technological pedagogical content knowledge: A framework for teacher knowledge. Teachers college record, 108(6), 1017-1054, En: h t t p : / / citeseerx.ist.psu.edu/viewdoc/ download?doi=10.1.1.523.3855\&rep=rep1\&type=pdf.

Nayebi, F., Desharnais, J. M., \&Abran,A. (2012). The state of the art of mobile application usability evaluation. In Electrical \& Computer Engineering(CCECE), 2012, 25th IEEE Canadian Conference on (pp. 1-4). IEEE. Recuperado de https:// ieeexplore.ieee.org/document/6334930/\#full-text-section

O’Loughlin, E. K., Sabiston, C. M., Dugas, E. N., \& O’Loughlin, J. L. (2015). The association between exercise behavior regulation and exergaming in adolescents. Journal of Physical Activity and Health, 12(3), 328-334.

Papadakis, S., \& Kalogiannakis, M. (2017). Mobile educational applications for children: what educators and parents need to know. International Journal of Mobile Learning and Organisation, 11(3), 256-277.

Papadakis, S., Kalogiannakis, M., \& Zaranis, N.(2017). Designing and creating an educational app rubric for preschool teachers. Education and Information Technologies, 22(6), 3147-3165.

Peng, H., Su, Y-J., Chou, C., \& Tsai, C-C. (2009) Ubiquitous knowledge construction: mobile learning re defined and a conceptual framework. Innovations in Education and Teaching International, 46(2), 171-183. doi:10.1080/ 14703290902843828

Phillips,A., Rodenbeck, M., \& Clegg, B. (2014). Apps for Physical Education: Teacher Tested, Kid Approved!, Strategies, 27(3), 28-31. doi:10.1080/ 08924562.2014.901047

Pimmer, C., \& Groehbiel, U. (2011). The affordances of social mobile media for boundary crossing. Integrating formal and informal learning. SSRE, 2013-2014.

Pourahmadi MR, Taghipour M, JannatiE, Mohseni-Bandpei MA, 
Ebrahimi Takamjani I, Rajabzadeh F. (2016) Reliability and validity of an iPhone ${ }^{\circledR}$ application for the measurement of lumbar spine flexion and extension range of motion. Peer J 4: e2355-e2355. doi.org/10.7717/peerj.2355

Prensky, M. (2011). Enseñar a nativos digitales. Madrid: SM.

Pulido, J.J., Sánchez, D., Sánchez-Miguel, P.A., González, I., \& García, T. (2016). Proyecto MÓVIL-ÍZATE: Fomento de la actividad física en escolares mediante las Apps móviles. RETOS. Nuevas Tendencias en Educación Física, Deporte y Recreación, (30), 3-8.

Rich,E., \& Miah,A. (2016). Mobile, wearableandingestiblehealth technologies: towards a critical research agenda. Health Sociology Review, 26(1), 84-97. doi:10.1080/ 14461242.2016.1211486

Robinson, K., \& Aronica, L. (2015). Escuelas creativas: La revolución que está transformando la educación. Barcelona: Grijalbo.

Romero-Franco, N., Jiménez-Reyes, P., Castaño-Zambudio, A., Capelo-Ramírez, F., Rodríguez-Juan,J.J., González-Hemández, J., Toscano-Bendala, F.J., Cuadrado-Peñafiel, V., \& BalsalobreFernández, C. (2016) Sprint performance and mechanical outputs computed with an iPhone app: Comparison with existing reference methods, European Journal of Sport Science, 17(4), 386-392. doi:10.1080/17461391.2016.1249031

Rosenthal, M., \& Eliason, S. (2015) »IHaveaniPad. Now What?» Using Mobile Devices in University Physical Education Programs,Journal of Physical Education, Recreation \& Dance, 86(6), 34-39. doi:10.1080/07303084.2015.1053636

Sandercock, G R. H.,Alibrahim, M., \&Bellamy, M.(2016). Media device ownership and media use: Associations with sedentary time, physical activity and fitness in english youth. Preventive Medicine Reports, 4, 162-168. doi:10.1016/ j.pmedr.2016.05.013

Schön, D. A. (1987). Jossey-Bass higher education series. Educating the reflective practitioner: Toward a new design for teaching and learning in the professions. San Francisco, CA, US: Jossey-Bass.

Schon, D. A. (1987). Educating the Reflective Practitioner. Toward a New Design for Teaching and Learning in the Professions. The Jossey-Bass Higher Education Series. Jossey-Bass Publishers, 350 Sansome Street, San Francisco, CA94104.

Sharples, M., \& Beale, R. (2003). A technical review of mobile computational devices. Journal of Computer Assisted Learning, 19(3), 392-395.

Silva,A.M., \& Montañez, L.F.(2019).Aprendizajepsicomotriz en el área de educación física, recreación y deportes mediado por el uso de software educativo. RETOS: nuevas tendencias en educación física, deporte y recreación, (36), 302309.

Smaldino, S. E., Russell, J. D., Heinich, R., \& Molenda, M. (2005). Instructional technology and media for learning (8th ed.). Upper Saddle River, NY: Pearson.

Song, Y. (2014). Bring your own device (BYOD)» for seamless science inquiry in a primary school. Computers and Education, 74,50-60. doi:10.1016/j.compedu.2014.01.005

Statistic Brain (2016). Mobile Phone App Store Statistics Physical Technology, In-App Purchase Revenue Statistics. En:http://www.statisticbrain.com/in-app-purchase-revenuestatistics/
Toffler, A. (1980). La Tercera Ola. Bogotá, Colombia, Plaza \& Janes Editores.

Traxler, J. (2007). Defining, Discussing and Evaluating Mobile Learning: The moving finger writes and having writ . . . IRRODEL.The International Review Of Research In Open And Distributed Learning, 8(2), 1-12. doi:http://dx.doi.org/ 10.19173/imodl.v8i2.346

Traxler, J. (2016). Inclusion in an age of mobility. Research in Learning Technology, (24), 1-13. doi:https://doi.org/10.3402/ rlt.v24.31372

Traxler, J. (2017). Mobile learning: the philosophical challenges, problems and implications of defining and theorising. Progressio: South African Journal for Open and Distance Learning Practice,39(1), 17-33. doi: 10.25159/0256$8853 / 1785$

Tuomi, I. (2018). The Impact of Artificial Intelligence on Learning, Teaching, and Education. Policies for the future, Eds. Cabrera, M., Vuorikari, R., \& Punie, Y., EUR 29442. Luxembourg: Publications Office of the EuropeanUnion. doi:10.2760/12297, JRC113226.

Turpo, O. (2018). La usabilidad pedagógica en la formación del profesorado: un estudio de caso. Revista ESPACIOS, 39(15). En: http://www.revistaespacios.com/a18v39n15/ 18391506.html

UNESCO (2016). Competencias y estándares TIC desde la dimensión pedagógica: una perspectiva desde los niveles de apropiación de las TIC en la práctica educativa docente. Recuperado de: http://www.unesco.org/new/fileadmin/ MULTIMEDIA/FIELD/Santiago/pdf/Competenciasestandares-TIC.pdfn

Walker, H. (2011). Evaluating the effectiveness of apps for mobile devices. Journal of Special Education Technology, 26(4), $59-63$.

White, D., \& Le Cornu, A. (2011). Visitors and Residents: Anew typology for online engagement. First Monday, 16(9). doi:https://doi.org/10.5210/fm.v16i9.3171

Williams, C. M., Caserta,A.J., \& Haines, T.P.(2013). The TiltMeter app is a novel and accurate measurement tool for the weight bearing lunge test. Journal of Science and Medicine in Sport, 16, 392-395. doi:10.1016/j.jsams.2013.02.001

Williamson, B. (2015). Algorithmic skin: Health-tracking technologies, personal analytics and the biopedagogies of digitized health and physical education. Sport, Education and Society, 20(1), 133-151.

Wyant, J., \& Baek, J.H. (2018) Re-thinking technology adoption in physical education, Curriculum Studies in Health and PhysicalEducation,1-15.doi:10.1080/25742981.2018.1514983

Yankov, L., \& Maion, S. (2014). Using iPads in Physical Education,ETEC510Wiki.En:http://etec.ctl.ubc.ca/510wiki/ Ipads_in_physical_education

Yu, H., Kulinna, P. H., \& Lorenz, K. A. (2018). An Integration of Mobile Applications into Physical Education Programs. Strategies, 31(3), 13-19. doi:10.1080/ 08924562.2018.1442275

Zhang, D., \&Adipat, B. (2005). Challenges, methodologies, and issues in the usability testing of mobile applications. International Journal of Human-Computer Interaction (18)3, 293-308. 